\title{
Nevoid melanoma: a rare and intriguing variant presenting as polypoidal mass on leg
}

\section{Anup Kumar Tiwary, Parul Singh}

\author{
Department of Dermatology, Subharti Medical College, Meerut, Uttar Pradesh, India \\ Corresponding author: Dr. Anup Kumar Tiwary, E-mail: anup07tunnu07@gmail.com
}

\begin{abstract}
Nevoid melanoma belongs to a rare and intriguing variant of melanoma which mostly present in elderly males as asymptomatic, single, variegated smooth plaques, verrucous nodule or polypoidal tumor usually affecting trunk and extremities. Clinically, the plaque forms can be misdiagnosed as seborrheic keratosis or papilloma. Nodular lesions mimicking benign dermal nevus and nodular melanoma should always be evaluated histopathologically. The risk of local recurrence and metastasis is always present depending upon the depth of invasion, hence management is no different than other more invasive forms of melanoma. Herein, we reported a female presenting with single, painless, blackish, firm, verrucous mass on right lower leg which was biopsied and confirmed on histopathology and immunostaining with HMB45 to be as nevoid melanoma.
\end{abstract}

Key words: Nevoid melanoma; Nest; Cord; Polypoid; Verrucous; Minimal-deviation melanoma

\section{INTRODUCTION}

Nevoid melanoma is a rare variant accounting for less than $1 \%$ of all cases of malignant melanoma. It was first described by Levene in 1980 as "verrucous and pseudonaevoid melanoma” [1]. In 1985, Schmoeckel et al. coined the term "naevoid malignant melanoma". It was also called by other names such as "minimal deviation melanoma' and 'small-cell melanoma' which are now considered separate histologic variants. It usually presents as solitary, brown/black, dome-shaped verrucous nodule or polypoidal growth mostly present on back and limbs [2]. Of note, due to its deceptive histologic simulation with benign dermal nevus, there is a potential diagnostic peril in the histologic assessment which may cause delay in diagnosis allowing its progression to higher stage ending in devastating outcome for the patient.

\section{CASE REPORT}

A 40-years-old female came to outpatient department of dermatology presenting with painless, mildly pruritic, solitary, blackish growth on right lower limb for more than 6 months. It started as a small pigmented papule which increased in size reaching up to the present size. On close cutaneous examination, lesion was seen as well-defined, black colored, polypoidal growth with mildly verrucous surface, present on medial side of right lower leg, measured of size $6 \mathrm{~cm} \times 7 \mathrm{~cm}$ (Fig. 1). There was no history of oozing or bleeding from the lesion. No history of preceding trauma, surgery or any preexisting dermatosis at the same site was noted. There was no known personal or familial history of cancer. Regional lymph nodes were not enlarged. General and systemic examination were also normal. Routine laboratory parameters were within normal limits. Before visiting our institution, she was given topical steroid formulations, oral steroids and oral antihistamines which relieved only itching with no effect on size. To confirm the diagnosis, we did incisional biopsy and sent the specimen for histopathologic examination.

On gross histopathology, section of specimen showed greyish-white to haemorrhagic area. Microscopically, it showed orthokeratotic stratum corneum with

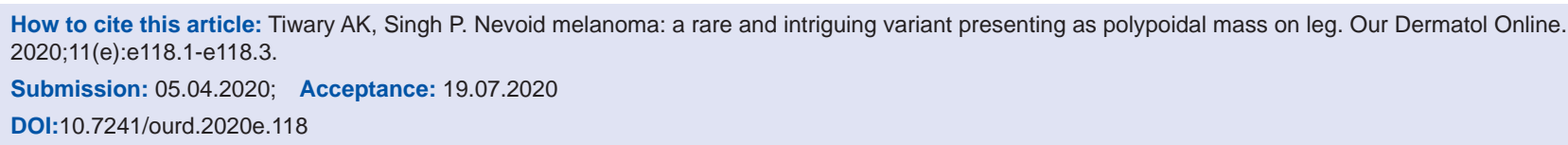




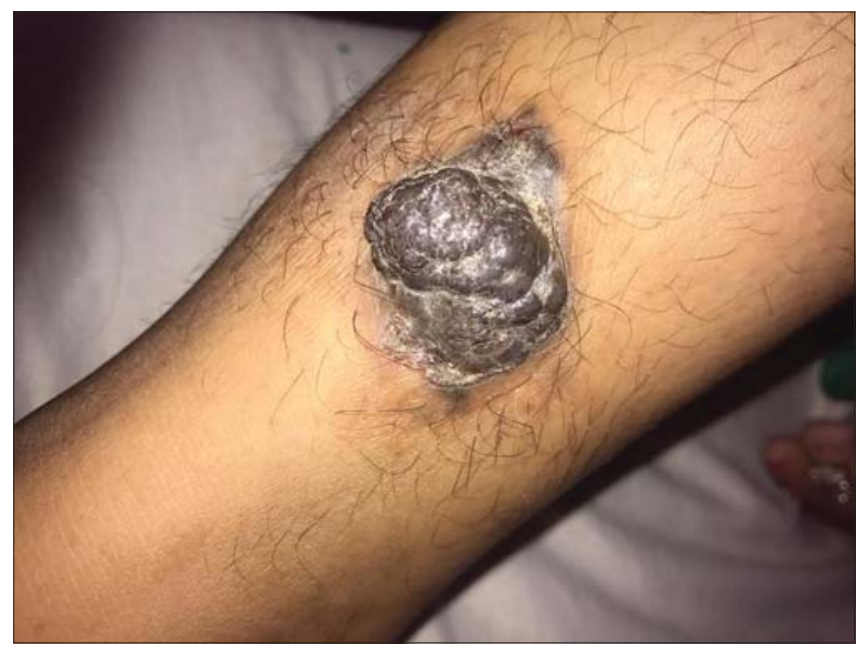

Figure 1: Well-defined blackish verrucous growth on right lower leg.

interspersed parakeratotic scale. Nests, cords and sheets of nevoid cells containing moderate amount of melanin and mixed inflammatory infiltrate of lymphocytes and neutrophils were present in papillary dermis with mild focal junctional activity (Fig. 2a). Cellular atypia, loss of orderly arrangement of nevoid nests (mostly at base), mild to moderate pleomorphism with prominent nucleoli, mitotic figures, lack of cellular maturation and infiltrative pattern were also seen (Fig. 2b). All these histopathologic features were in favour of nevoid melanoma. Immunostaining with HMB-45 was also performed which came out to be positive. Thus, it was diagnosed as nevoid melanoma. There was no metastasis seen on positron emission tomography (PET) scan and patient was referred to oncology department for appropriate management.

\section{DISCUSSION}

Nevoid melanomas clinically present mostly as asymptomatic (unless ulcerated or secondarily infected), black or brown papillomatous plaques/ domed nodules of size $>1 \mathrm{~cm}$ in diameter with smooth borders, or polypoidal growth with verrucous surface and irregular borders due to intraepidermal, radial growth phase [2]. The most common sites are trunk and limbs. There is male preponderance with 2:1 ratio and age group ranges from 5th-7th decade [3]. Local recurrence and metastasis to regional lymph nodes is not uncommon, having been reported in up to $50 \%$ cases with subsequent mortality of at least $25 \%$.

Histopathologic features of nevoid melanoma is often diagnostic. At low power, dome-shaped or polypoid surface contour is seen showing expansile

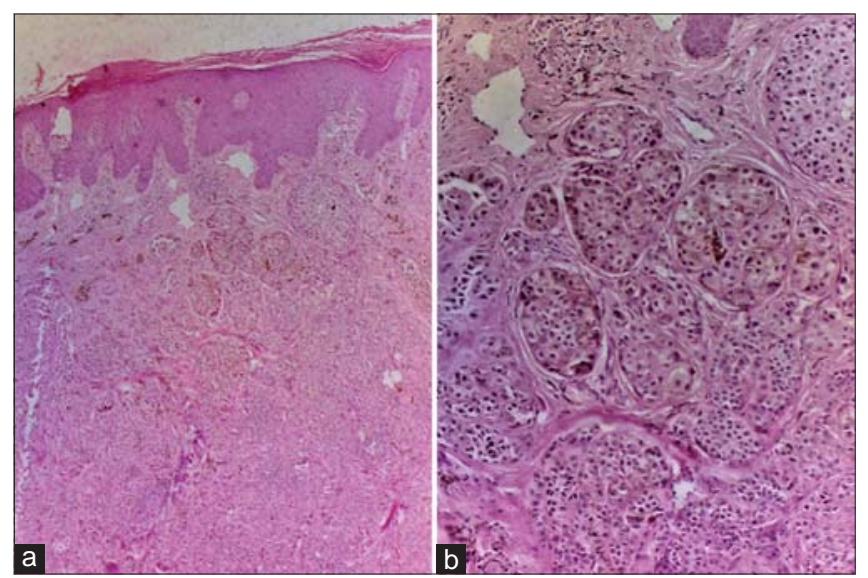

Figure 2: (a) Irregularly acanthotic epidermis, mild papillomatosis, small nests, short cords and sheets of neoplastic melanocytes (H \& E, $\times 100$ ), (b) Expansile nodular proliferation of neoplastic melanocytes in papillary dermis showing cellular atypia, pleomorphism, prominent nucleoli and lack of cellular maturation at base $(H \& E, \times 200)$.

dermal nodules distorting the papillary dermis due to melanocytic proliferation. High power magnification reveals superficial nests, short cords and parallel theques of neoplastic melanocytes. In the verrucous variant, the superficial nests can be quite large and confluent. Dermal component may extend up to the superficial reticular dermis [4]. Junctional activity is uncommon, mostly observed in verrucoid forms of nevoid melanoma. Fine melanin dusts may be seen in some cases. The size of the neoplastic melanocytes becomes smaller with descent in the dermis (loss of maturation) but atypical cytological features such as increased nuclear-to-cytoplasmic ratio, nuclear membrane irregularity, hyperchromasia, mitotic activity and conspicuous nucleoli are invariably present at all depths. Immunohistochemical studies using markers such as HMB-45 (antibody to melanosomal gpl00), Mibl (an antibody to Ki-67, a surrogate marker for proliferation), cyclins A, B, Dl/D3, pl6, WT-1, metallothionein and antibody to leptin receptor can potentially aid in confirming the doubtful histopathological diagnosis [5].

The differential diagnosis includes minimal-deviation melanoma, nodular melanoma, melanoma arising in a pre-existing dermal nevus, benign congenital and acquired dermal nevus, combined nevus, inverted type A (clonal) nevus and metastatic melanoma [4]. Neoplastic features including prominent nucleoli, hyperchromasia, increased nuclear-to-cytoplasmic ratios and mitotic activity are not seen in the benign dermal nevus. High-grade nuclear atypia and intraepidermal melanocytic atypia are characteristics of nodular melanoma. The minimal-deviation 
melanoma shows relatively monotypic cells with mild atypia and low mitotic figures in contrast to nevoid melanoma. Melanoma arising in a dermal nevus has deeper extension, usually up to the subcutaneous layer unlike in nevoid variant. Combined nevus (e.g. an intradermal and cellular blue nevus) differs from nevoid melanoma by larger melanocytes of the blue nevus component at the deeper location and reduced HMB45 immunoreactivity. An inverted type A nevus can be differentiated by larger nevus cells at the base of the lesion and lack of nuclear and cytologic atypia. Metastatic melanoma can be ruled out by strict dermal involvement with minimal epidermal component, history of prior melanoma and multiple satellite lesions [4].

Early diagnosis and scanning for regional and distant metastasis is mandatory to reduce the morbidity and mortality. According to American Joint Committee on Cancer (AJCC) staging criteria, stage I has around 95\% 5 -year survival and stage IV with median survival of 6 to 9 months. In general, thin melanomas $(<1.0 \mathrm{~mm})$ are best excised with a $1 \mathrm{~cm}$ margin and those between 1-4 mm need a $2 \mathrm{~cm}$ margin. Melanoma thicker than $4 \mathrm{~mm}$ should have wider margins $(2-3 \mathrm{~cm})$.

\section{CONCLUSION}

All cases presenting with dark-colored, slowly progressing, verrucous or polypoidal mass with no preceding skin lesion must be biopsied for histopatholgic examination to rule out melanoma. As nevoid melanoma is rare and closely simulate the microscopic features of benign acquired nevus, a meticulous pathologic assessment is warranted before concluding a diagnosis to avoid inappropriate management.

\section{Consent}

The examination of the patient was conducted according to the Declaration of Helsinki principles.

The authors certify that they have obtained all appropriate patient consent forms. In the form the patient(s) has/have given his/her/ their consent for his/her/their images and other clinical information to be reported in the journal. The patients understand that their names and initials will not be published and due efforts will be made to conceal their identity, but anonymity cannot be guaranteed.

\section{REFERENCES}

1. Levene A. On the histological diagnosis and prognosis of malignant melanoma. J Clin Pathol. 1980;33:101-24.

2. Zembowicz A, McCusker M, Chiarelli C, Dei Tos AP, Granter SR, Calonje E, et al. Morphologic analysis of nevoid melanoma: a study of 20 cases with a review of the literature. Am J Dermatopathol. 2001;23:167-75.

3. Theodosiou G, Johansson I, Hamnerius N, Svensson A. Naevoid malignant melanoma: a diagnosis of a naevus that you later regret. Acta Derm Venereol. 2017;9:97:745-6.

4. Magro CM, Crowson AN, Mihm MC Jr. Unusual variant of malignant melanoma. Mod Pathol. 2006;19:41-70.

5. Mc Nutt NS. Nevoid malignant melanoma. Rev Esp Patol. 1999;32:451-2.

Copyright by Anup Kumar Tiwary, et al. This is an open access article distributed under the terms of the Creative Commons Attribution License, which permits unrestricted use, distribution, and reproduction in any medium, provided the original author and source are credited.

Source of Support: Nil, Conflict of Interest: None declared. 